\title{
Perspectives in systems biology
}

\section{Soumen Roy}

Published online: 16 February 2014

(C) Springer Science+Business Media Dordrecht 2014

For this special issue, I have hopefully been able to assemble an assortment of diverse and interesting articles reflecting the wide range of phenomena where systems biology approaches are being successfully applied. This issue has nine papers containing contributions from twenty six researchers.

DevRS is an important two component system in Mycobacterium tuberculosis. Through their model, Bandyopadhyay et al. propose that DevR controlled gene expression follows a specific pattern which efficiently describes other DevR mediated gene expression. Using tools of information theory the authors calculate the molecular efficiency of the system and show that it is close to the maximum limit of isothermal efficiency.

Basu et al. consider an interesting kind of network from the link-weight distribution of microRNA co-target networks of many different species which are universal up to scaling. The number of cell types which is a measure of complexity is found to be proportional to the scale-factors. They propose universal features of these networks and show that this universality splits if one considers distributions of number of common targets of three or more miRNAs. These distributions for different species can be collapsed onto two distinct sets of universal functions, implying that the species which appeared earlier in history of evolution seem to possess a different measure of complexity compared to species that appeared later.

With the advent of new genomic technologies, methods which use additional algorithms for Reverse Vaccinology (RV), have increased potential to reduce likelihood of

S. Roy $(\bowtie)$

Bose Institute, 93/1 Acharya PC Roy Road, Kolkata 700 009, India

e-mail: soumen@jcbose.ac.in undesirable features including allergenicity and immune cross reactivity to host. Chaudhuri et al. assemble a database called MycobacRV which includes collection of known vaccine candidates and a set of predicted vaccine candidates identified from the whole genome sequences of many human pathogenic mycobacterium strains and species. These predicted vaccine candidates are the adhesins and adhesin-like proteins obtained using rather stringent cutoffs. Subsequently, these protein sequences were studied through publicly available algorithms and analysis of Enhanced RV information by the authors yielded several nonredundant, most probable vaccine candidates. The authors have also created an online server regarding this work at http://mycobacteriarv.igib.res.in

Dasgupta proposes a basis for entropy enthalpy compensation in biochemical and metabolic networks. He discusses the possibility of non-existence of positive efficiency of a chemically driven process and proposes to circumvent this by deriving an appropriate definition of macroscopic force using the local balance equations. The method provides an account of how reactive pathways are coupled. Further, he points out that this approach also provides a thermodynamic rationale for Bergmann's rule.

Ghosh et al. address the question of identifying biochemical routes and conduct a comparison of six different weighting schemes to derive node and edge weights for metabolic graphs, where weights reflect various kinetic/ thermodynamic parameters as well as abundances inferred from transcriptomic data. Using a network of of carbohydrate metabolism, they identify kinetic parameter derived weighting schemes which fare best. The methods can be used in the analysis for metabolic networks from any species and for comparing condition-specific networks.

Priya et al. examine pathway genes from different microbial genomes, belonging to a wide range of groups, to assess inter- and intra-genic variations in these groups. 
They describe these genetic and genomic variations in tryptophan biosynthetic pathway genes in different microorganisms to show the similarities across organisms and compare the same genes across different organisms to explore the possible variability which might likely arise due to horizontal gene transfers. Studies like these could be stepping stones for moving from single gene evolution to pathway evolutionary studies.

Raman et al. revisit the organisation of protein networks and the centrality-lethality hypothesis, which states that nodes with higher centrality in a network are more likely to produce lethal phenotypes on removal as compared to nodes with lower centrality. They consider protein-protein networks of high-confidence interactions for a diverse set of organisms whose essentiality information was obtained by them from the Database of Essential Genes. They then assess the relationship between centrality measures and lethality on these networks by computing network parameters such as degree, betweenness centrality, closeness centrality and pairwise disconnectivity indices. Essential nodes in these disassortative networks have a significantly higher average degree and betweenness centrality compared to the average node and the centrality-lethality hypothesis seems to holds good for a large number of organisms, with certain limitations. They also find that betweenness centrality may be significantly correlated with essentiality but measures like closeness centrality and pairwise disconnectivity are not really high for essential nodes.

The choice of type 1 or type 2 pathway is an important question in the systems biology of apoptosis signaling. Raychaudhuri and Raychaudhuri use in-silico approaches to elucidate the role of membrane proximal signaling module in this choice. Their results provide crucial mechanistic insights into the formation of membrane proximal death-inducing-signaling-complex (DISC) signalosome and caspase 8 activation. Increased concentration of death ligands is shown by them to correlate with increased type 1 activation. They also analyze the caspase 6 mediated system level feedback activation of apoptosis signaling and it's role in the type 1/type 2 choice. Their results clarify the basis of cell-to-cell stochastic variability in apoptosis activation and indicate ramifications for therapies of neurodegenerative disorders and cancer.

Somvanshi and Venkatesh treat a disease diagnosis problem analogous to fault diagnosis problems in engineering systems. They review the application of engineering methodologies to address human diseases from systems biology perspective. Their review highlights potential networks and modeling approaches used for analyzing human diseases with specific illustrations for cancer and diabetes. They put forth a concept of cell-tohuman framework comprising five modules (data mining, networking, modeling, experimentation and validation) for addressing human physiology and diseases based on a paradigm of system level analysis. Their review emphasizes the importance of multi-scale biological networks and their modeling and analyses for drug target identification and design of efficient therapies.

\section{In this issue:}

Bandyopadhyay A, Biswas S, Maity AK and Banik SK (2014) Analysis of DevR regulated genes in Mycobacterium tuberculosis

Basu M, Bhattacharyya NP and Mohanty PK (2014) Universality splitting in distribution of number of miRNA cotargets

Chaudhuri R, Kulshreshtha D, Raghunandanan MV and Ramachandran $S$ (2014) Integrative immunoinformatics for mycobacterial diseases in $\mathrm{R}$ platform

Dasgupta AK (2014) Finite time thermodynamic coupling in a biochemical network

Ghosh S, Baloni P, Vishveshwara S and Chandra N (2014)

Weighting schemes in metabolic graphs for identifying biochemical routes

Priya VK, Sarkar S and Sinha S (2014) Evolution of tryptophan biosynthetic pathway in microbial genomes: a comparative genetic study

Raman K, Damaraju N and Joshi GK (2014) The organisational structure of protein networks: revisiting the centrality-lethality hypothesis

Raychaudhuri S and Raychaudhuri SC (2014) Death ligand concentration and the membrane proximal signaling module regulate the type 1/type 2 choice in apoptotic death signaling

Somvanshi PR and Venkatesh KV (2014) A conceptual review on systems biology in health and diseases: from biological networks to modern therapeutics 